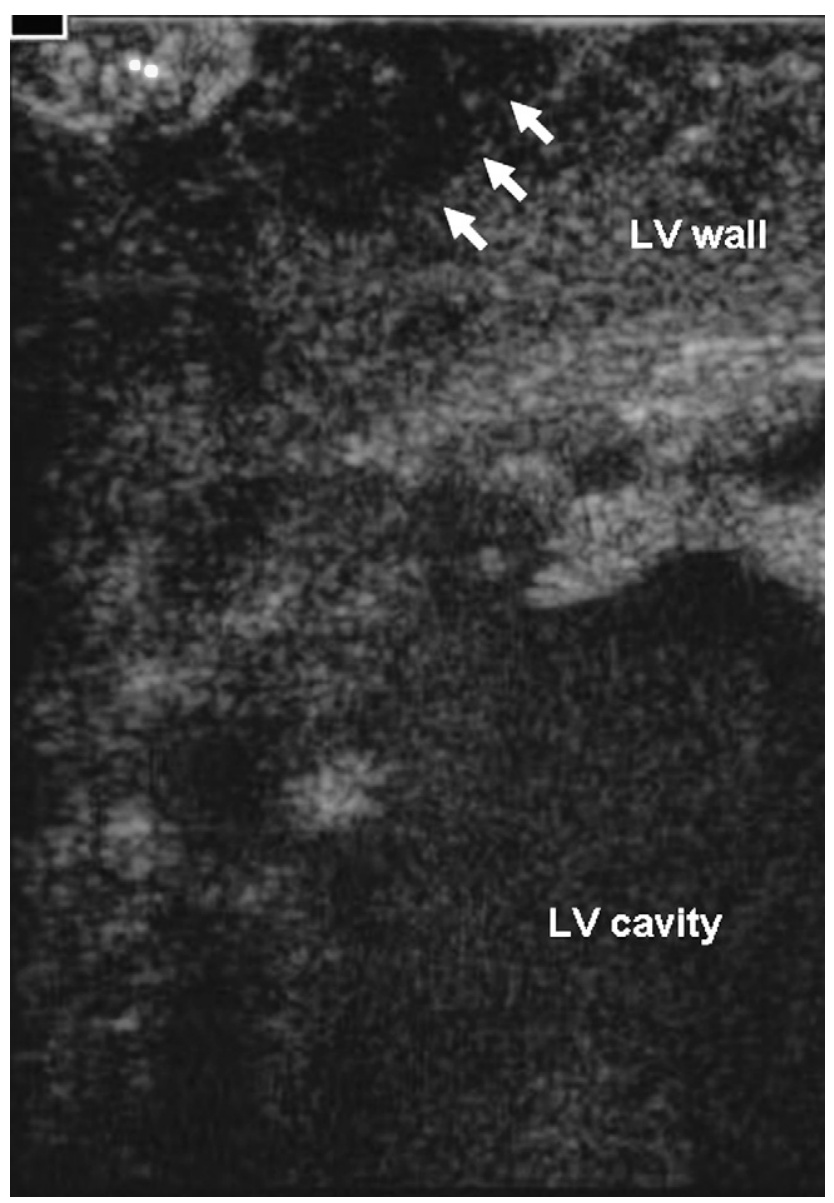

FIGURE 2. Arrows indicate the abscess in the anterior left ventricular wall on epicardial echocardiography.

during cardiac surgery or autopsy. ${ }^{3}$ The reported incidence during autopsy ranges between $0.2 \%$ and $1.5 \% .^{1,2}$ They usually occur in the setting of IE with Gram-positive cocci ${ }^{1,2}$ and some- times in infarcted myocardium when circulating microorganisms get seeded in the region. ${ }^{4}$ Our patient, who presented with chest pain and electrocardiogram changes consistent with acute coronary syndrome (ACS), was incidentally found to have $K$ pneumoniae myocardial abscess during CABG.

Maslow et $\mathrm{al}^{4}$ have reported the only other case of myocardial abscess due to $K$ pneumoniae. Their patient had $K$ pneumoniae urosepsis associated with acute myocardial infarction, resulting in myocardial abscess and papillary muscle rupture. The diagnosis was made during surgery for mitral valve replacement. The patient improved after therapy with cefotaxime; however, cardiac rupture occurred on postoperative day 6 .

There are no guidelines for treating $K$ pneumoniae infection involving the heart but a 6 -week course of aztreonam and ciprofloxacin proved effective in our patient. She is still doing well at the end of 1 year. To the best of our knowledge, this patient is the only documented survivor of a $K$ pneumoniae myocardial free wall abscess. A cardiac computed tomography or magnetic resonance imaging scan for patients with high clinical suspicion of a cardiac infection is a good test for diagnosing as well as localizing a myocardial free wall abscess. This might increase the incidence of detecting myocardial abscesses in living subjects, rather than it being predominantly an autopsy finding.

\section{References}

1. Flaxman N. Myocardial abscess. JAMA. 1943;122:804.

2. Sanson J, Slodki S, Gruhn JG. Myocardial abscesses. Am Heart J. 1963; 66:308-8.

3. Rohmann S, Seifert T, Erbel R, et al. Identification of abscess formation in nativevalve infective endocarditis using transesophageal echocardiography: implications for surgical treatment. Thorac Cardiovasc Surg. 1991;39:273-80.

4. Maslow MJ, Reitano JM, Schnall HA, Shah AT, Simberkoff MS, Rahal JJ. Myocardial abscess due to Klebsiella pneumoniae complicating acute infarction. Am J Med Sci. 1984;287:58-60.

\title{
Sex reversal and hypoplastic left heart syndrome
} \author{
Andrew M. Atz, MD, ${ }^{\text {a }}$ Charleston, SC
}

Michael J. Walsh, MD, ${ }^{\mathrm{a}}$ Elizabeth T. Walsh, MD, ${ }^{\mathrm{b}}$ Yaw Appiagyei-Dankah, MD, ${ }^{\mathrm{b}}$ and
The prognosis of congenital heart disease, especially in its most complex forms, is complicated when associated with extracardiac anomalies. Meacham syndrome, or PAGOD syndrome (pulmonary tract and pulmonary artery hypoplasia, agonadism, omphalocele, diaphragmatic defect, and dextrocardia) is a constellation of genital, cardiac, and pulmonary malformations that carries a poor prognosis. We report the case of a patient with sex reversal and hypoplastic left heart syndrome without congenital diaphragmatic hernia who has survived to Fontan completion and is doing well. 


\section{CLINICAL SUMMARY}

The patient, whose condition was diagnosed prenatally with fetal ultrasonography, was found at birth to have hypoplasia of the left ventricle and the mitral and aortic valves, with coarctation of the aorta and proximal stenoses of the branch pulmonary arteries. A Norwood procedure with the Sano modification was performed on day 6 after birth. After 2 days of persistent hypoxemia, the patient was taken back to the operating room, where the Sano shunt was replaced with a modified Blalock-Taussig shunt. At the age of 5 months, the patient underwent bidirectional superior cavopulmonary anastomosis. At 3.5 years of age, the patient underwent successful extracardiac conduit Fontan completion with fenestration. Her post-Fontan course to the age of 4.5 years has been uncomplicated.

Initial evaluation revealed normal female external genitalia and no obvious dysmorphology. The presurgical workup for complex congenital heart disease at our institution includes chromosomal analysis, as well as ultrasonography of the head and kidneys. As part of this routine evaluation, the patient's karyotype was revealed to be $46, \mathrm{XY}$. This was confirmed by repeated testing and by demonstration of the presence of the $S R Y$ gene. Subsequent pelvic ultrasonography failed to demonstrate a uterus or gonads. Laboratory evaluation showed no evidence of congenital adrenal hyperplasia, androgen insensitivity syndrome, or a central pituitary process.

Birth weight was $2.575 \mathrm{~kg}$ (3rd percentile). Thereafter, weight and height were consistently below the 3 rd percentile, although following a stable isopleth. The trend has continued through the most recent clinic visit at 4 years 7 months. The mechanism of this persistent growth failure is unclear. Arginine and clonidine stimulation testing did not suggest isolated growth hormone deficiency.

\section{DISCUSSION}

Chromosomal abnormalities, as well as noncardiac anomalies, have been identified as independent risk factors for increased surgical morbidity and mortality in single-ventricle palliation. ${ }^{1}$ This combination also leads to increased rates of termination of pregnancy and increased levels of parental stress for those surviving the first stage of palliation. Tworetzky and colleagues ${ }^{2}$ documented improved survival of infants with hypoplastic left heart syndrome who had the condition prenatally diagnosed relative to those without early diagnosis. It follows that the prenatal identification of noncardiac anomalies would afford affected infants a better chance at survival. Karyotyping at the time of diagnosis of significant congenital heart disease has therefore become standard at our institution. When an anatomic diagnosis is made by fetal echocardiography, amniocentesis is recommended.
This case also illustrates the interplay between abnormal karyotype and fetal growth with respect to surgical planning. Many chromosomal abnormalities are characterized by poor fetal growth. Lower birth weight is associated with worse outcomes in neonates undergoing initial palliation for single-ventricle physiology. ${ }^{1}$ As we try to elucidate further the causes of increased surgical mortality associated with complex congenital heart disease, we must address the way low birth weight, as it relates to chromosomal abnormalities, puts these infants at higher risk.

Although the association of chromosomal abnormalities and complex congenital heart disease has been well studied with respect to prognosis and surgical morbidity and mortality, implications of specific abnormalities have often not been evaluated. Survival of patients with both hypoplastic left heart syndrome and Turner syndrome is markedly reduced relative to those patients with hypoplastic left heart syndrome alone. ${ }^{3}$ According to a multi-institutional study, most patients with trisomy 13 or 18 can survive palliative or corrective heart surgery ${ }^{4}$; however, this study did not include patients with single-ventricle lesions. More investigation into the survival of patients with specific chromosomal abnormalities and complex congenital heart disease is needed.

Previous reports of Meacham syndrome have been associated with extremely poor prognosis. ${ }^{5}$ It is noteworthy that most of these patients had significant loss of lung volume secondary to diaphragmatic hernias. This case illustrates many important features in the evaluation and management of a patient with sex reversal, growth failure, and hypoplastic left heart syndrome. It is notable that despite numerous previously reported risk factors, this child has done well and has survived multistage surgical reconstruction to Fontan completion. Finally, we are reminded of the need for close endocrine follow-up with a patient such as this, including evaluation for the presence of any gonadal tissue and other disturbances of the hormonal axis.

\section{References}

1. Stasik CN, Gelehrter S, Goldberg CS, Bove EL, Devaney EJ, Ohye RG. Current outcomes and risk factors for the Norwood procedure. $J$ Thorac Cardiovasc Surg. 2006; 131:412-7.

2. Tworetzky W, McElhinney DB, Reddy VM, Brook MM, Hanley FL, Silverman NH. Improved surgical outcome after fetal diagnosis of hypoplastic left heart syndrome. Circulation. 2001;103:1269-73.

3. Reis PM, Punch MR, Bove EL. van de Ven CJ. Outcome of infants with hypoplastic left heart and Turner syndromes. Obstet Gynecol. 1999;93:532-5.

4. Graham EM, Bradley SM, Shirali GS, Hills CB, Atz AM. Effectiveness of cardiac surgery in trisomies 13 and 18 (from the Pediatric Cardiac Care Consortium). Am J Cardiol. 2004;93:801-3.

5. Meacham LR, Winn KJ, Culler FL, Parks JS. Double vagina, cardiac, pulmonary, and other genital malformations with 46,XY karyotype. Am J Med Genet. 1991;41: 478-81. 